\title{
Reflexionseigenschaften von Windenergieanlagen im Funkfeld von Funknavigations- und Radarsystemen
}

\author{
S. Sandmann, S. Divanbeigi, and H. Garbe \\ Institut für Grundlagen der Elektrotechnik und Messtechnik, Leibniz Universität Hannover, Deutschland \\ Correspondence to: S. Sandmann (sandmann@geml.uni-hannover.de)
}

Received: 15 December 2014 - Revised: 26 March 2015 - Accepted: 26 March 2015 - Published: 3 November 2015

\begin{abstract}
Kurzfassung. Die hier behandelte Untersuchung befasst sich mit den Störungen des elektrischen Feldes einer Doppler Very High Frequency Omnidirectional Radio Range Navigationsanlage (DVOR) in der Gegenwart von Windenergieanlagen (WEA). Hierfür wird die Feldstärke auf 25 konzentrischen Kreisbahnen, sog. Orbit Flights verschiedener Höhen und mit verschiedenen Radien rund um die DVOR-Anlage numerisch simuliert. Insbesondere werden die Einflüsse diverser Parameter der WEA wie deren Anzahl, Position, Rotorwinkel, Turmhöhe und Rotordurchmesser auf die Feldverteilung herausgestellt, sowie die Anwendbarkeit der Simulationsmethode Physical Optics (PO) durch Vergleich der Simulationsergebnisse mit denen der Multi Level Fast Multipol Method (MLFMM) untersucht.
\end{abstract}

\section{Einleitung}

Die durch die Umgestaltung der Energieversorgung in Richtung erneuerbarer Energien bedingte Zunahme der Windenergieanlagen (WEA) und deren immer größere Ausmaße verursachen immer häufiger elektromagnetische Störungen beim Betrieb von Radar- und Navigationsanlagen ${ }^{1}$. Dies ist u. a. darin begründet, dass eine WEA sowohl durch ihre Ausmaße als auch durch die eingesetzten Baumaterialien als Reflektor für die betreffenden Frequenzen fungiert und damit eine potentielle Störquelle darstellt. Um die Möglichkeit einer solchen Wechselwirkung zwischen WEA und Navigationsanlage vorherzusagen, ist es daher notwendig, das entsprechende Störpotential bereits vor dem Bau der WEA einschätzen zu können. Hierfür bietet sich die numerische Si-

\footnotetext{
${ }^{1}$ Gallardo-Hernando et al. (2011) und http://www. wind-energie.de/sites/default/files/attachments/page/ arbeitskreis-luftverkehr-und-radar/20131107-bwe-umfrage-radar. pdf
}

mulationen der elektromagnetischen Wellenausbreitung an. Die in dieser Untersuchung behandelte Doppler Very High Frequency Omnidirectional Radio Range Navigationsanlage (DVOR) übermittelt Flugzeugen eine Winkelinformation zu Lokalisierungszwecken. Wie in Abb. 1a dargestellt, wird der betreffende Winkel $\alpha$ am Ort der DVOR-Antenne azimutal aufgespannt durch den Schnitt der auf die Horizontalebene projezierten Verbindungslinien zwischen Antenne und magnetisch Nord bzw. Antenne und Flugzeug. Hierfür strahlt die DVOR-Anlage das azimutabhängige Signal im Frequenzbereich um $112 \mathrm{MHz}$ mit einem hochachsensymmetrischen Richtdiagramm nach Abb. $1 \mathrm{~b}$ in horizontaler Polarisation ab. Abhängig von seinem Standort empfängt das Flugzeug mit dem Signal die darin entsprechend kodierte Winkelinformation, die durch einen Auswertealgorithmus aus dem Signal im Flugzeugempfänger ermittelt wird.

Diese kann jedoch bei nicht idealen Vorraussetzungen für die Ausbreitung der elektromagnetischen Wellen prinzipiell durch diverse Effekte verfälscht werden (Yamamoto und Nagaoka, 1989; Wakabayashi et al., 1998). Um die sehr komplexen Zusammenhänge zwischen einzelnen Feldparametern und dem entstehenden Winkelfehler durch Zerlegung in einzelne Signalkomponenten erfassen zu können und dabei den Aufwand der Simulationen auf ein vertretbares Maß zu reduzieren, beschränkt sich diese Untersuchung auf die Beeinflussung der Trägerfeldstärke der DVOR-Anlage. Die Beeinflussung wird als Differenz der Feldstärkepegel mit und ohne WEA als Störobjekt und insbesondere in Abhängigkeit von diversen Parametern wie Anzahl, Position, Rotorwinkel, Turmhöhe und Rotordurchmesser der WEA dargestellt. Es ist hingegen hier nicht das Ziel, den Fehler auf die Flugführungsgröße „Azimutwinkel“ konkret zu bestimmen. Dies bleibt weiteren Arbeiten vorbehalten. 


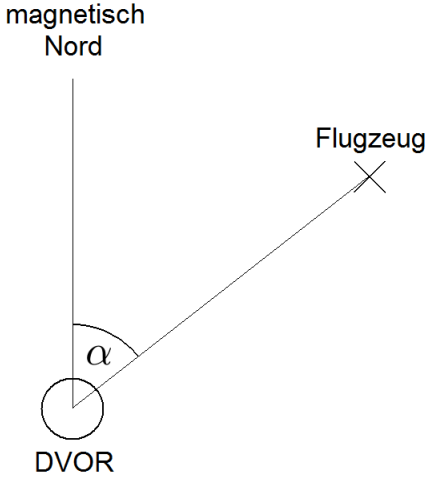

a)

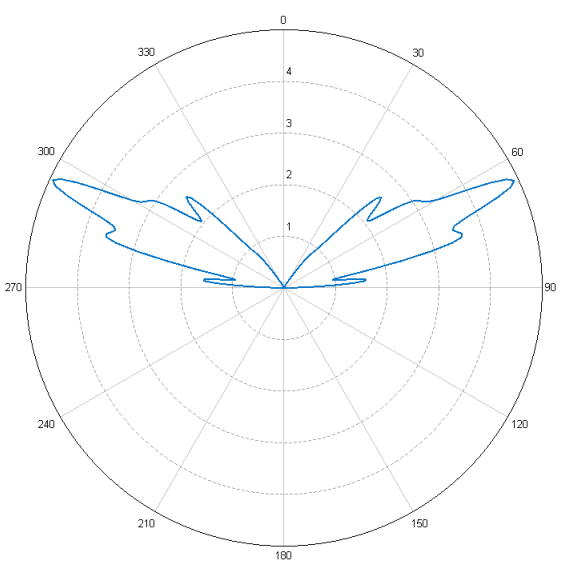

b)

Abb. 1. Veranschaulichung der übertragenen Winkelinformation und vertikales Richtdiagramm des Trägersignals einer DVOR-Anlage.

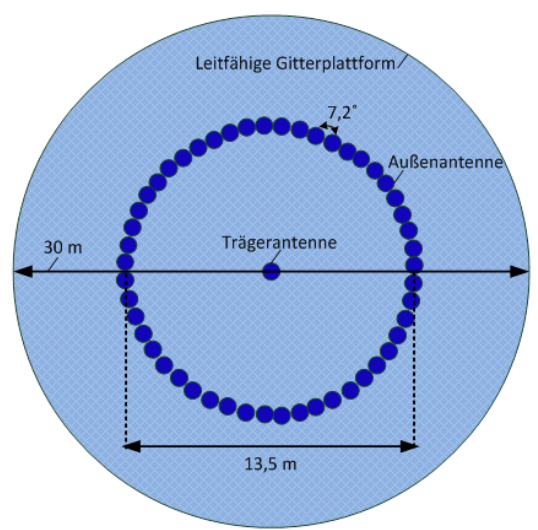

a)

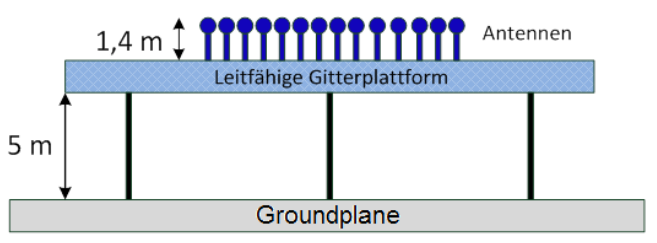

b)

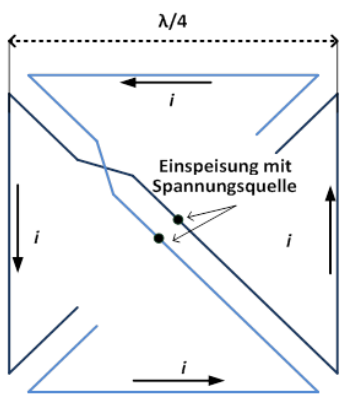

c)

Abb. 2. Schematische Darstellung einer DVOR-Anlage und einer Alford-Loop-Antenne.

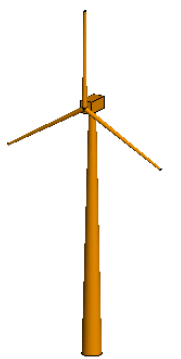

a)

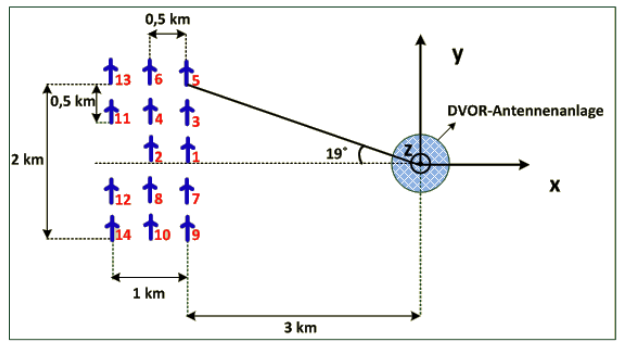

b)

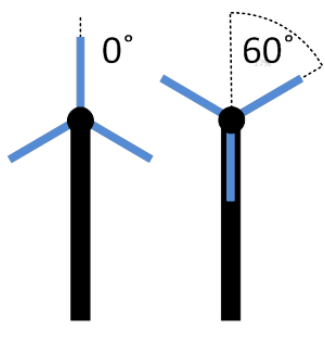

c)

Abb. 3. (a) Modell der simulierten WEA vom Typ ENERCON E82. (b) Illustration der Platzierung einzelner WEA in einem Park. (c) Definition zweier Rotorwinkel. 


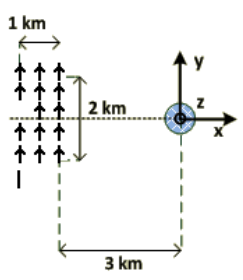

(a)

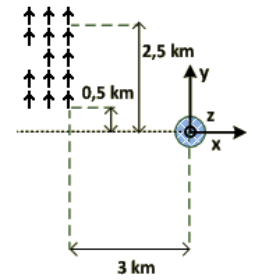

(b)

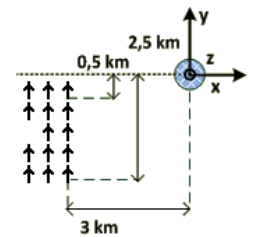

(c)

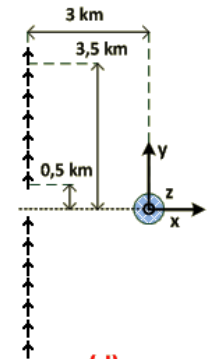

(d)

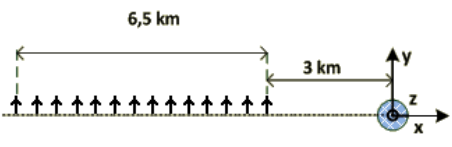

(e)

Abb. 4. Darstellung verschiedener Formationen eines WEA-Parks.

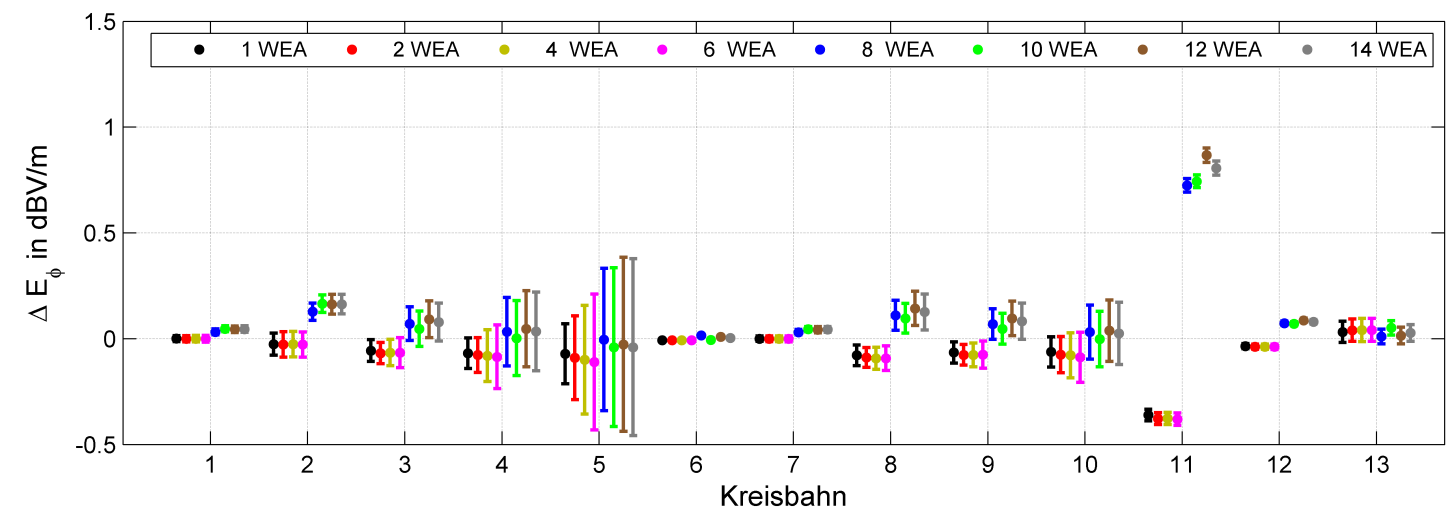

Abb. 5. MLFMM-Simulationsergebnisse der Kreisbahnen 1 bis 13 bei Variation der WEA-Anzahl.

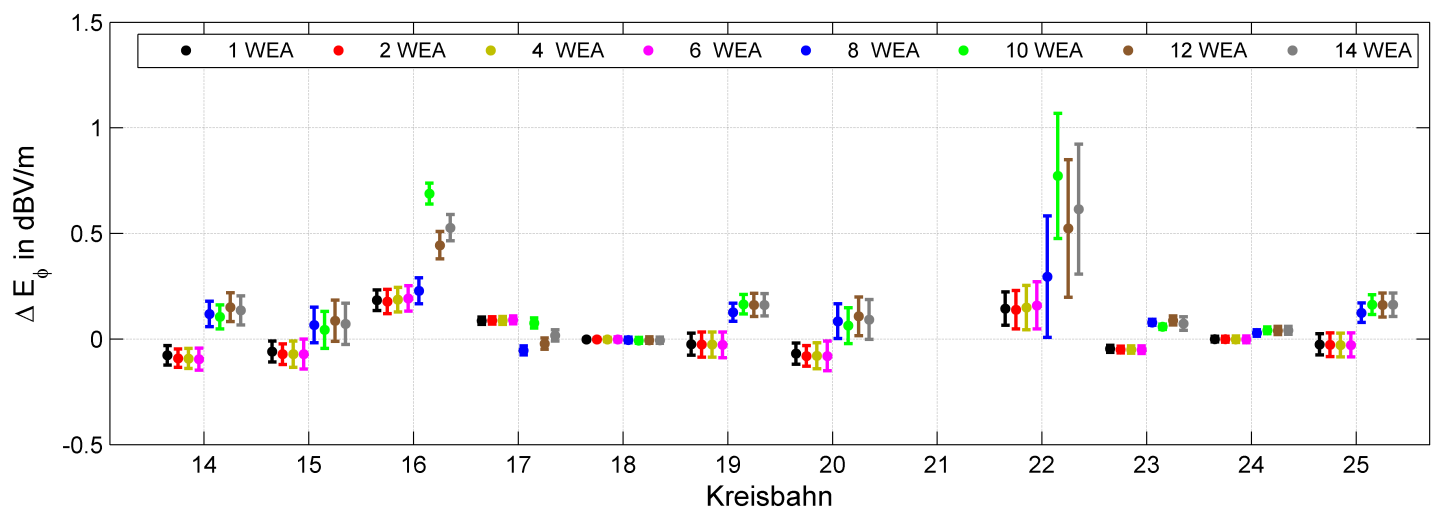

Abb. 6. MLFMM-Simulationsergebnisse der Kreisbahnen 14 bis 25 bei Variation der WEA-Anzahl. Die Werte der 21. Kreisbahn sind aus Darstellungsgründen nicht abgebildet $\left(\left|M_{21}\right| \leq 6,2 \mathrm{dBV} \mathrm{m}^{-1} ;\left|S_{21}\right| \leq 1,47 \mathrm{dBV} \mathrm{m}^{-1}\right)$. 
Das verwendete Simulationstool „FEKO“ bietet unterschiedliche Verfahren zur Behandlung von elektrisch großen Objekten an (http://feko.info). Für das vorliegende Simulationsszenario, das sich durch hohe Frequenzen bei elektrisch großen Objekten auszeichnet, eignet sich die Multi Level Fast Multipol Method (MLFMM). Da der hierbei entstehende Arbeitsspeicherbedarf, der mit der Zahl der Unbekannten $N$ proportional zu $N \cdot \log N$ ansteigt (van Tonder und Jakobus, 2005), bei der Simulation von Szenarien im Ausmaß von mehreren WEA die vorhandenen Kapazitäten rasch überschreitet, bietet sich neben der mathematisch strengen Simulationsmethode MLFMM auch die optische Näherungsmethode PO an, deren Arbeitsspeicherbedarf weitaus geringer ist (Jakobus und Meyer, 1996). Die Zulässigkeit dieser Vereinfachung wird anhand von Abweichungen der Ergebnisse durch Vergleichssimulationen untersucht.

Nach der Beschreibung der Simulationsszenarios im nächsten Abschnitt, werden deren Ergebnisse im Abschnitt 3 diskutiert. Abschließend werden die wichtigsten Erkenntnisse der Untersuchung in einem Fazit herausgestellt.

\section{Simulationsszenario}

Das grundlegende Simulationsszenario besteht aus einer DVOR-Anlage als Strahlungsquelle im Ursprung des Koordinatensystems über einer ideal leitenden Groundplane. Wie Abb. $2 a$ und $b$ veranschaulichen, ist die DVOR-Anlage ihrerseits aus einer leitfähigen Gitterplattform und 51 AlfordLoop-Antennen (vgl. Abb. 2c) aufgebaut, von denen 50 kreisförmig um eine im Mittelpunkt der Plattform platzierte Antenne angeordnet sind.

Als aktiv strahlendes Element dient dabei lediglich die Alford-Loop-Antenne im Zentrum der Anlage, da das hier betrachtete Trägersignal vollständig über diese Antenne abgestrahlt wird. Deren Speisung wurde dabei mit $112 \mathrm{MHz}$ und $250 \mathrm{~V}$ gerade so gewählt, dass das Abstrahlverhalten in etwa den realen Gegebenheiten entspricht. Die übrigen, kreisförmig angeordneten Antennen, die für die Abstrahlung der richtungsabhängigen Signalkomponenten zuständig sind, sind am Antennenfußpunkt durch eine komplexe Impedanz angepasst abgeschlossen. Das achsensymmetrische Abstrahldiagramm der so betriebenen DVOR-Anlage ist in Abb. 1b dargestellt.

Als reflektierendes Störobjekt befindet sich in einem Abstand von $3 \mathrm{~km}$ auf der negativen $x$ Achse eine Konstellation von WEA-Modellen nach Abb. 3a die dem Typ E82 der Firma Enercon nachempfunden sind. Diese werden nach den Abb. 3b, c und 4 hinsichtlich mehrerer Parameter variiert: Anzahl der WEA, Rotorwinkel, Turmhöhe, Rotordurchmesser und Formation des WEA-Parks.

Als Ergebnis der einzelnen Simulationen werden mit dem Programm FEKO die Feldstärkewerte in horizontaler Polarisation auf 25 Kreisbahnen in verschiedenen Höhen und mit verschiedenen Radien (vgl. Tabelle 1) mit der Metho- de MLFMM berechnet. Die Mittelpunkte sämtlicher Bahnen fallen dabei mit der zentralen Achse der DVOR-Anlage zusammen. Zur Auswertung werden die Differenzen der Feldstärkepegel des gestörten Falls (Referenzsimulation, ohne WEA, Index $R$ ) und des nicht gestörten Falls (Vergleichssimulation, mit WEA, Index $V$ ) nach Gl. (1) gebildet.

$$
\begin{aligned}
\Delta E_{\phi}(\theta) & =20 \log \sqrt{\operatorname{Re}\left\{E_{\phi, V}(\theta)\right\}^{2}+\operatorname{Im}\left\{E_{\phi, V}(\theta)\right\}^{2}} \\
& -20 \log \sqrt{\operatorname{Re}\left\{E_{\phi, R}(\theta)\right\}^{2}+\operatorname{Im}\left\{E_{\phi, R}(\theta)\right\}^{2}}
\end{aligned}
$$

Des Weiteren wird durch Vergleichssimulationen die Anwendbarkeit der für elektrisch sehr große Objekte entwickelten asymptotischen Methode PO untersucht, die bei geringfügigen Einbußen der Ergebnisgenauigkeit eine deutliche Reduktion des Arbeitsspeicherbedarfs verspricht (Jakobus und Meyer, 1996). Hierbei werden sowohl die WEA als auch die Gitterfläche der DVOR-Anlage mit der PO-Full Ray Tracing (PO-FT) berechnet.

Während die mathematisch strenge MLFMM als Referenzmethode herangezogen wird, werden hierfür die Berechnungen mit der Methode PO als Vergleichsmethode in Gl. (1) eingesetzt.

\section{Simulationsergebnisse}

Die Abb. 5 und 6 stellen die Feldstärkedifferenzen der Kreisbahnen nach Gl. (1) durch ihre Mittelwerte und Standardabweichungen der jeweils 360 Messpunke dar.

Deutlich zu erkennen ist eine leicht steigende Tendenz der Standardabweichungen der meisten Kreisbahnen mit steigender Anzahl der WEA und steigenden Radien der Kreisbahnen nach Tabelle 1. Des Weiteren sind auch erhöhte Werte auf den Kreisbahnen 11, 16, 21 und $22 \mathrm{zu}$ verzeichnen. Die Ursache dieser Zunahme liegt im Abstrahlverhalten der DVOR-Antenne. Wie in Abb. 1b dargestellt, strahlt die DVOR-Anlage hauptsächlich bei einem Elevationswinkel von etwa $25^{\circ}$, während sie bei einem Winkel von über $45^{\circ}$ einen sogenannten ,cone of silence“ aufweist, in dem das DVOR-Signal im Flugbetrieb nicht ausgewertet wird. In diesem Bereich und damit auf den Kreisbahnen 11, 16, 21 und 22 ist das Verhältnis des von den WEA gestreuten Feldanteils zum direkten Feldanteil der DVOR-Anlage daher höher als bei den restlichen Kreisbahnen, was sich durch entsprechend erhöhte Feldstärkedifferenzen äußert. Die Werte der 21. Kreisbahn wurden dabei aus Darstellungsgründen nicht abgebildet, da sie mit einem Betrag des Mittelwertes $M_{21}$ von bis zu $6,2 \mathrm{dBV} \mathrm{m}^{-1}$ und einer Standardabweichung $S_{21}$ von bis $\mathrm{zu} 1,47 \mathrm{dBV} \mathrm{m}^{-1}$ stark von den Werten der anderen Kreisbahnen abweichen.

Bei einem Vergleich der Ergebnisse der Simulationsmethode MLFMM mit den in den Abb. 7 und 8 dargestellten Ergebnissen der Methode PO lässt sich ein qualitätiv ähnliches Verhalten feststellen, wobei die Wertebereiche allgemein um 
Tabelle 1. Radien und Höhen der 25 Kreisbahnen zur Berechnung der Feldstärkewerte.

\begin{tabular}{|c|c|c|c|c|c|}
\hline & $\begin{array}{c}r_{5} \\
=10 \mathrm{NM} \approx 18520 \mathrm{~m}\end{array}$ & $\begin{array}{c}r_{4} \\
=5 \mathrm{NM} \approx 9260 \mathrm{~m}\end{array}$ & $\begin{array}{c}r_{3} \\
=3 \mathrm{NM} \approx 5556 \mathrm{~m}\end{array}$ & $\begin{array}{c}r_{2} \\
=1 \mathrm{NM} \approx 1852 \mathrm{~m}\end{array}$ & $\begin{array}{c}r_{1} \\
=0,5 \mathrm{NM} \approx 926 \mathrm{~m}\end{array}$ \\
\hline$h_{5}$ & Kreisbahn & Kreisbahn & Kreisbahn & Kreisbahn & Kreisbahn \\
\hline$=10000 \mathrm{ft} \approx 3048 \mathrm{~m}$ & 25 & 24 & 23 & 22 & 21 \\
\hline$h_{4}$ & Kreisbahn & Kreisbahn & Kreisbahn & Kreisbahn & Kreisbahn \\
\hline$=5000 \mathrm{ft} \approx 1524 \mathrm{~m}$ & 20 & 19 & 18 & 17 & 16 \\
\hline$h_{3}$ & Kreisbahn & Kreisbahn & Kreisbahn & Kreisbahn & Kreisbahn \\
\hline$=3500 \mathrm{ft} \approx 1067 \mathrm{~m}$ & 15 & 14 & 13 & 12 & 11 \\
\hline$h_{2}$ & Kreisbahn & Kreisbahn & Kreisbahn & Kreisbahn & Kreisbahn \\
\hline$=2000 \mathrm{ft} \approx 610 \mathrm{~m}$ & 10 & 9 & 8 & 7 & 6 \\
\hline$h_{1}$ & Kreisbahn & Kreisbahn & Kreisbahn & Kreisbahn & Kreisbahn \\
\hline$=1000 \mathrm{ft} \approx 305 \mathrm{~m}$ & 5 & 4 & 3 & 2 & 1 \\
\hline
\end{tabular}

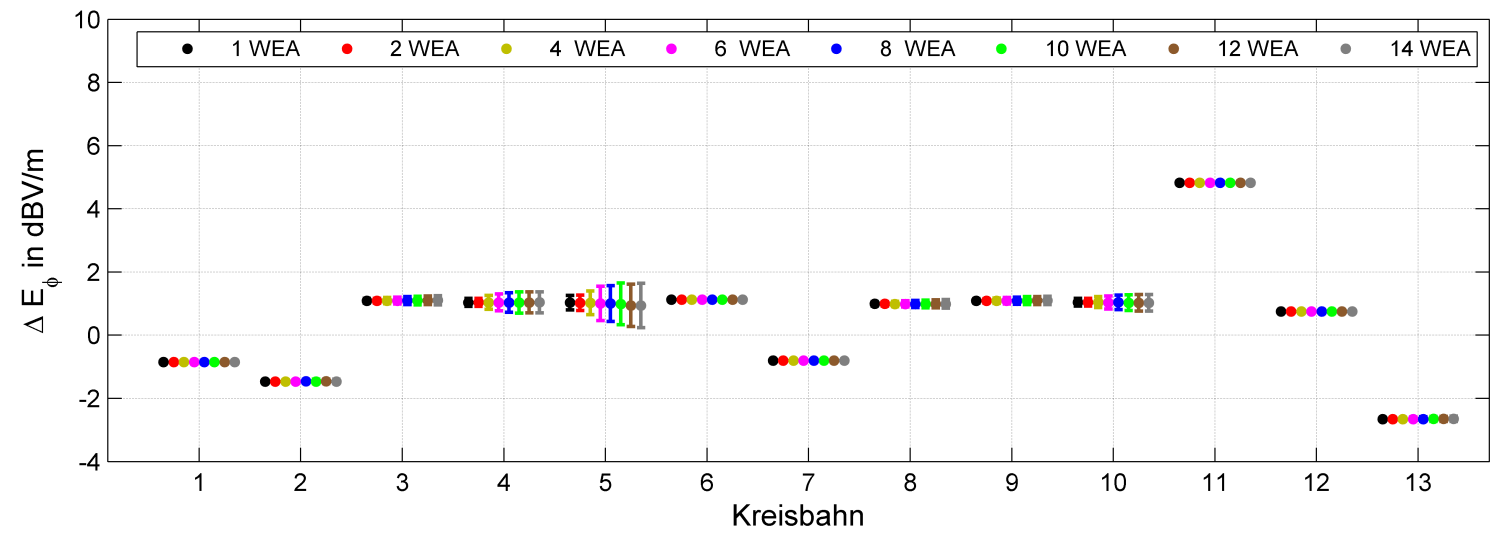

Abb. 7. PO-Simulationsergebnisse der Kreisbahnen 1 bis 13 bei Variation der WEA-Anzahl.

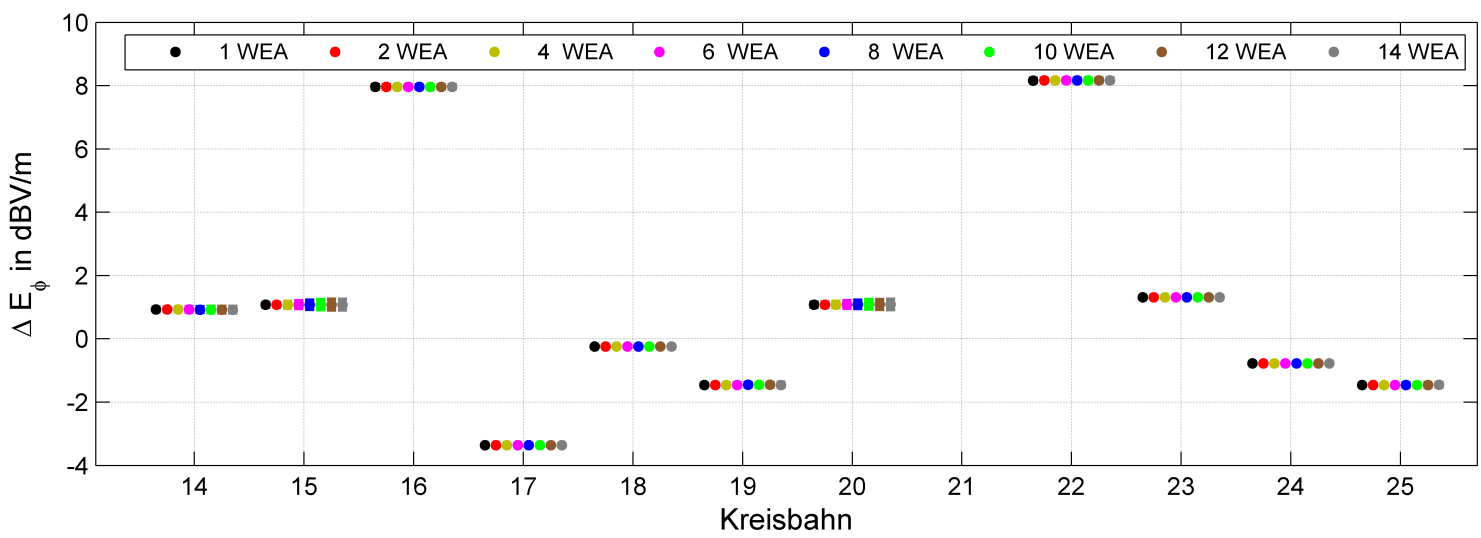

Abb. 8. PO-Simulationsergebnisse der Kreisbahnen 14 bis 25 bei Variation der WEA-Anzahl. Die Werte der 21. Kreisbahn sind aus Darstellungsgründen nicht abgebildet $\left(\left|M_{21}\right| \leq 26,7 \mathrm{dBV} \mathrm{m}^{-1} ;\left|S_{21}\right| \leq 0,41 \mathrm{dBV} \mathrm{m}^{-1}\right)$. 

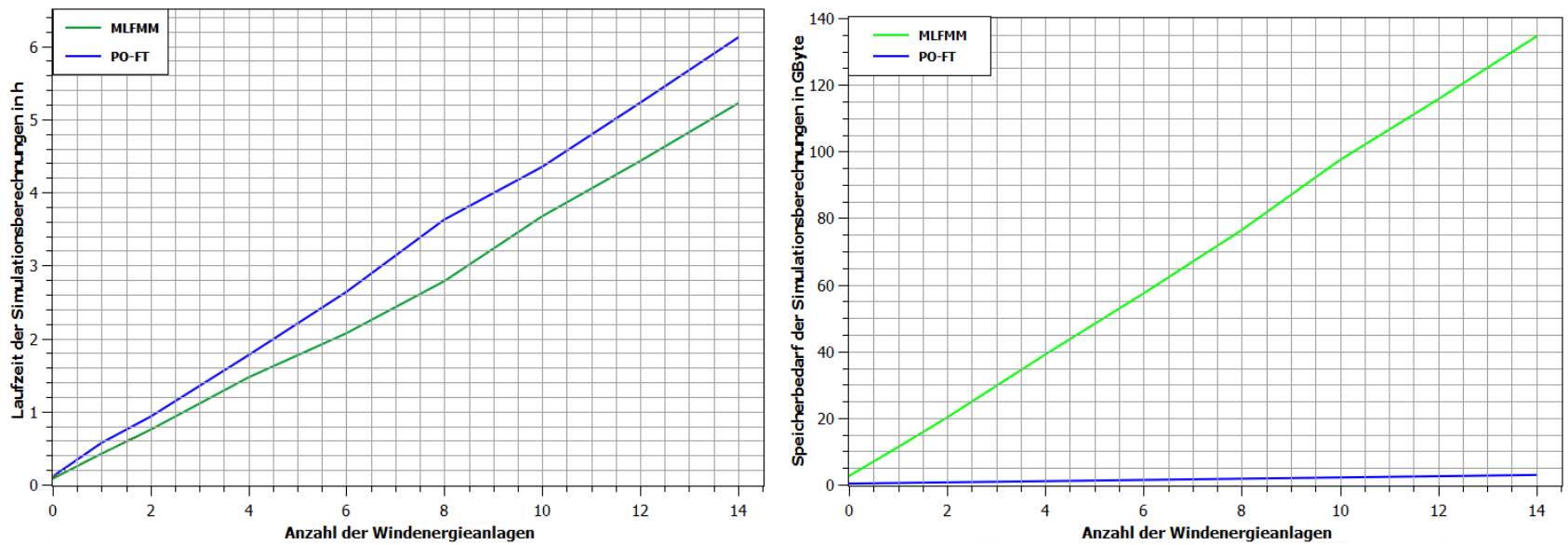

Abb. 9. Gegenüberstellung des Ressourcenbedarfs der Simulationsmethoden MLFMM und PO in Abhängigkeit der Anzahl an WEA.

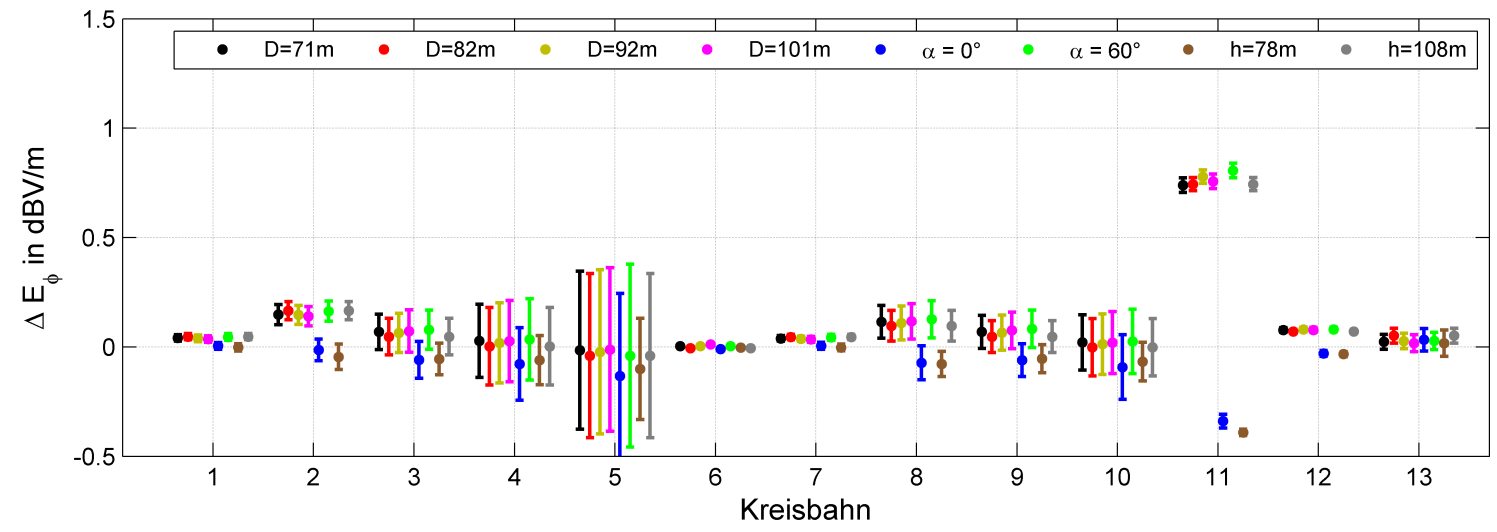

Abb. 10. MLFMM-Simulationsergebnisse der Kreisbahnen 1 bis 13 bei Variation des Rotordurchmessers $D$, des Rotorwinkels $\alpha$ und der Turmhöhe $h$.

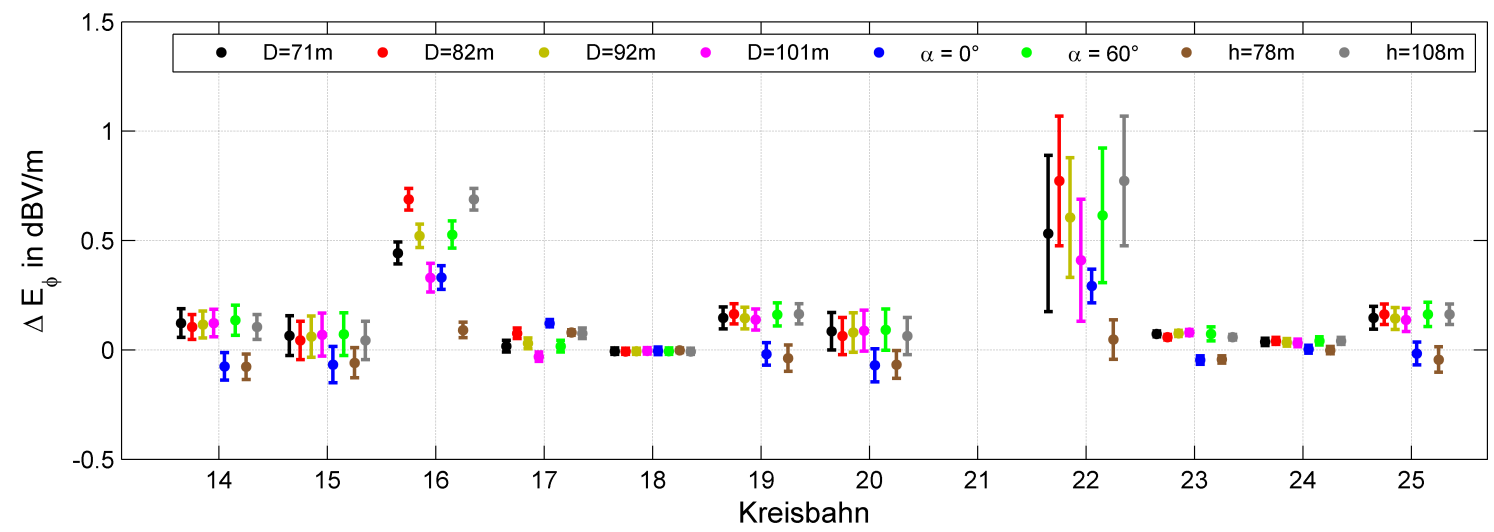

Abb. 11. MLFMM-Simulationsergebnisse der Kreisbahnen 14 bis 25 bei Variation des Rotordurchmessers $D$, des Rotorwinkels $\alpha$ und der Turmhöhe $h$. Die Werte der 21. Kreisbahn sind aus Darstellungsgründen nicht abgebildet $\left(\left|M_{21}\right| \leq 6,44 \mathrm{dBV} \mathrm{m}^{-1} ;\left|S_{21}\right| \leq 1,51 \mathrm{dBV}^{-1}\right)$. 


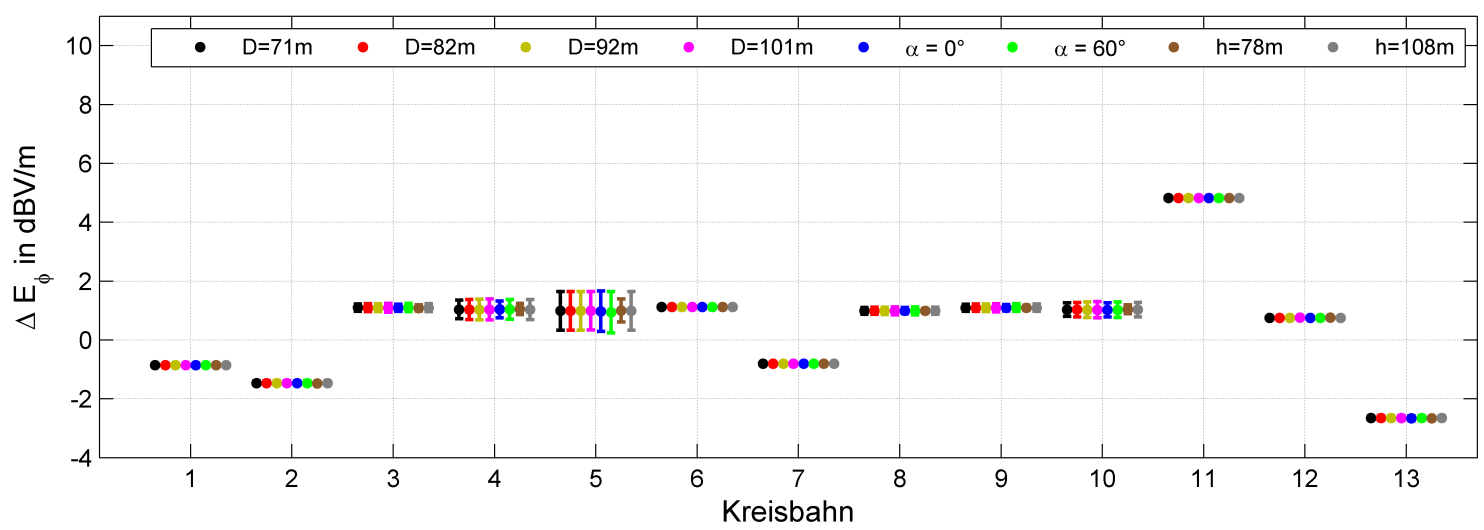

Abb. 12. PO-Simulationsergebnisse der Kreisbahnen 1 bis 13 bei Variation des Rotordurchmessers $D$, des Rotorwinkels $\alpha$ und der Turmhöhe $h$.

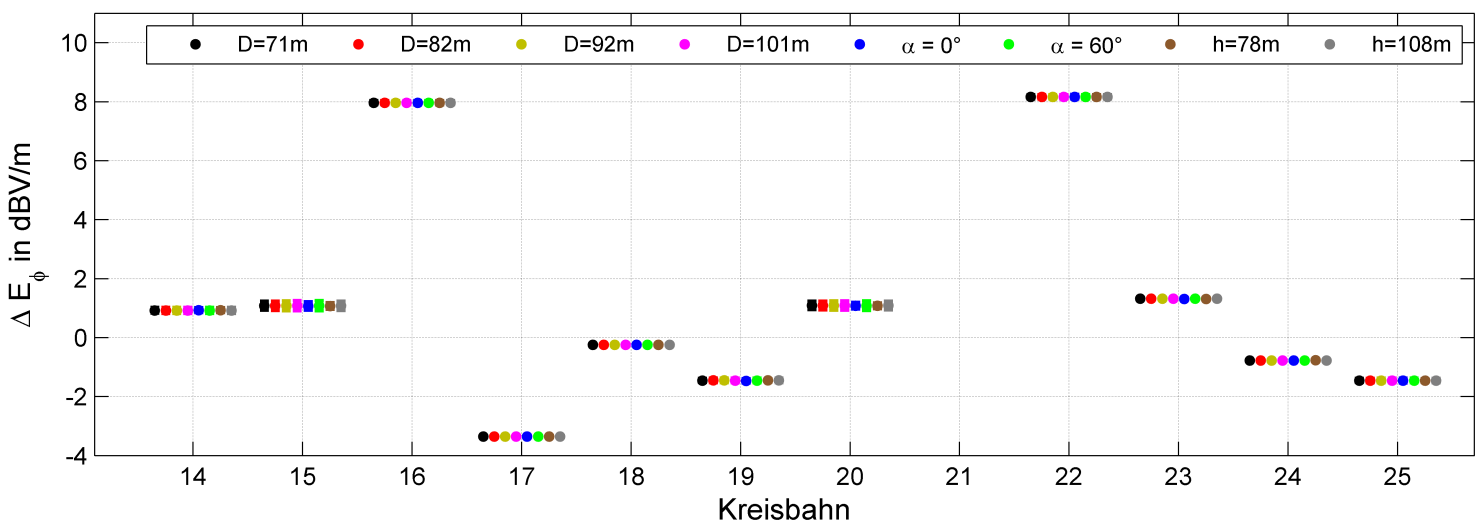

Abb. 13. PO-Simulationsergebnisse der Kreisbahnen 14 bis 25 bei Variation des Rotordurchmessers $D$, des Rotorwinkels $\alpha$ und der Turmhöhe $h$. Die Werte der 21. Kreisbahn sind aus Darstellungsgründen nicht abgebildet $\left(\left|M_{21}\right| \leq 26,69 \mathrm{dBV} \mathrm{m}^{-1} ;\left|S_{21}\right| \leq 0,42 \mathrm{dBV}^{-1}\right)$.

etwa den Faktor 10 größer ausfallen. Somit lässt sich festhalten, dass die Methode PO gegenüber der MLFMM bei dem betrachteten Simulationsszenario eine weitaus geringere Ergebnisgenauigkeit liefert.

Der Vorteil dieser Methode zeigt sich im Ressourcenverbrauch. Wie Abb. 9 verdeutlicht, verbraucht die Methode PO bei leicht erhöhter Laufzeit lediglich etwa $3 \%$ des Arbeitsspeicherbedarfs der Methode MLFMM.

Die Einflüsse des Rotordurchmessers, der Turmhöhe und des Rotorwinkels nach Abb. 3c bei der Anwesenheit von 14 WEA nach Abb. $3 b$ lassen sich den Abb. 10 und 11 für die Simulationsmethode MLFMM entnehmen. Allgemein lässt sich ein nur geringer Einfluss der 3 Parameter von unter $0,5 \mathrm{dBV} \mathrm{m}^{-1}$ auf die Feldstärkedifferenzen erkennen, der auch keine ausgeprägten Tendenzen vermuten lässt. Auch bei diesen Simulationsergebnissen lassen sich die größten Störungen im Bereich des „cone of silence“ auf den Kreisbahnen 11, 16, 21 und 22 verzeichnen. Die Beträge der Mittelwerte der 21. Kreisbahn erreichen dabei Werte von bis zu $6,4 \mathrm{dBV} \mathrm{m}^{-1}$, während die größte Standardabweichung etwa $1,5 \mathrm{dBV} \mathrm{m}^{-1}$ beträgt.
Die entsprechenden Ergebnisse der Simulationsmethode PO sind in den Abb. 12 und 13 dargestellt. Analog zu den bereits behandelten Untersuchungen lässt sich auch hier ein um etwa den Faktor 10 erhöhter Wertebereich der Feldstärkedifferenzen bei ansonsten qualitativ ähnlichem Verhalten gegenüber der Simulationsmethode MLFMM verzeichnen.

Die Simulationsergebnisse bei Variation der Formation eines WEA-Parks bestehend aus 14 WEA sind in den Abb. 14 und 15 dargestellt. Sämtliche Abweichungen außer den Kreisbahnen im „,cone of silence“ können mit Werten deutlich unterhalb $0,5 \mathrm{dBV} \mathrm{m}^{-1}$ als sehr gering angenommen werden. Innerhalb des „,cone of silence“ zeigen sich die bereits bekannten Überhöhungen der Feldstärkedifferenzen. Ferner weisen sämtliche Bahnen leichte Auffälligkeiten bei der Formation $e$ ) auf. Aufgrund der fluchtenden Anordnung der WEA mit der DVOR-Anlage zeigen sich besonders bei dieser Anordnung größere Feldstärkedifferenzen im elektromagnetischen Schatten der WEA.

Dies ist für die 5. Kreisbahn exemplarisch in Abb. 16 verdeutlicht, in der sich die fluchtenden WEA beim Azimutwinkel von $180^{\circ}$ befinden. 


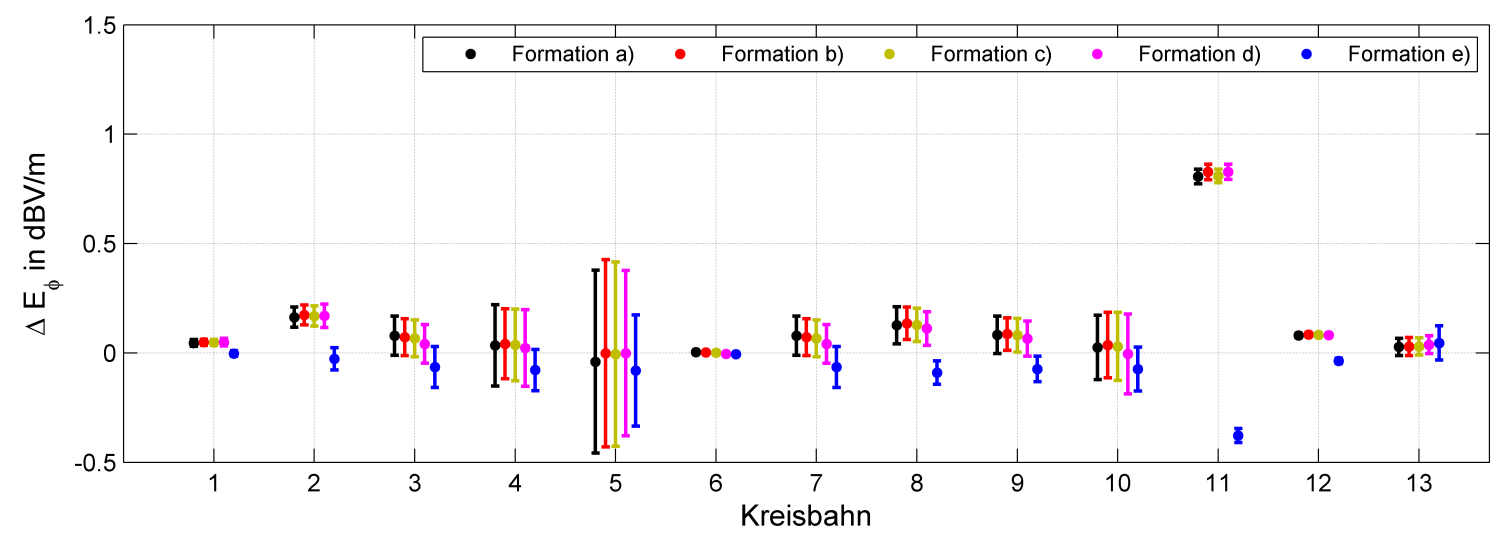

Abb. 14. MLFMM-Simulationsergebnisse der Kreisbahnen 1 bis 13 bei Variation der WEA-Park-Formation.

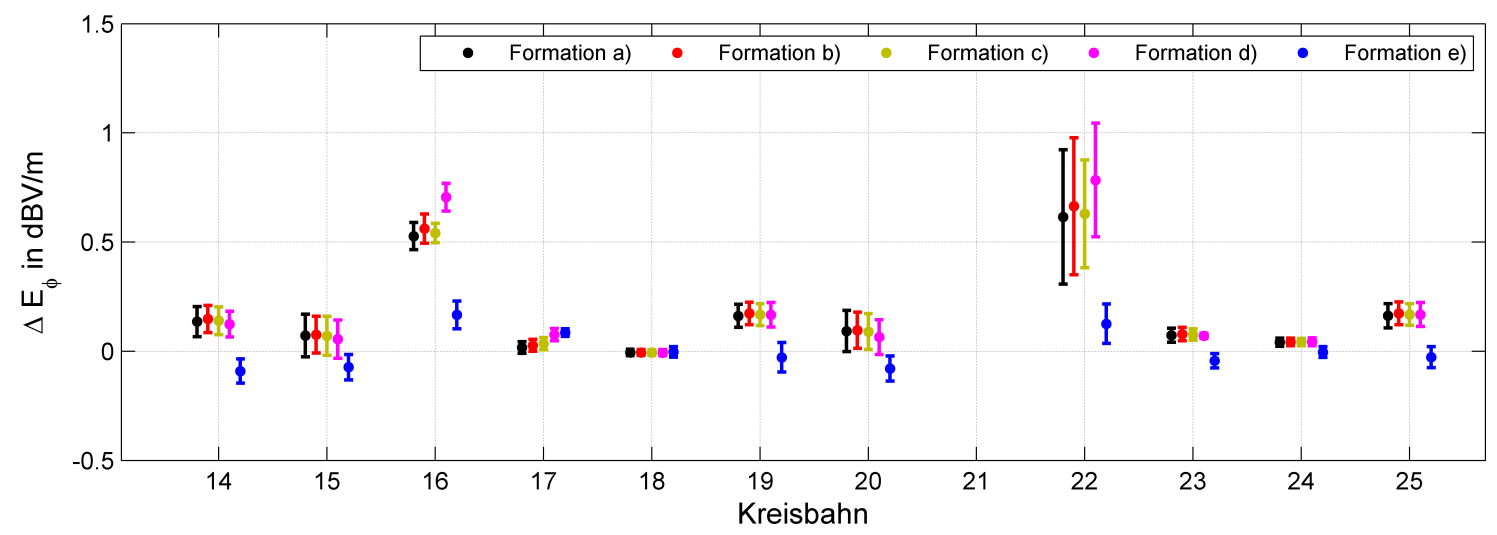

Abb. 15. MLFMM-Simulationsergebnisse der Kreisbahnen 14 bis 25 bei Variation der WEA-Park-Formation. Die Werte der 21. Kreisbahn sind aus Darstellungsgründen nicht abgebildet $\left(\left|M_{21}\right| \leq 4,98 \mathrm{dBV} \mathrm{m}^{-1} ;\left|S_{21}\right| \leq 1,47 \mathrm{dBV} \mathrm{m}^{-1}\right)$.

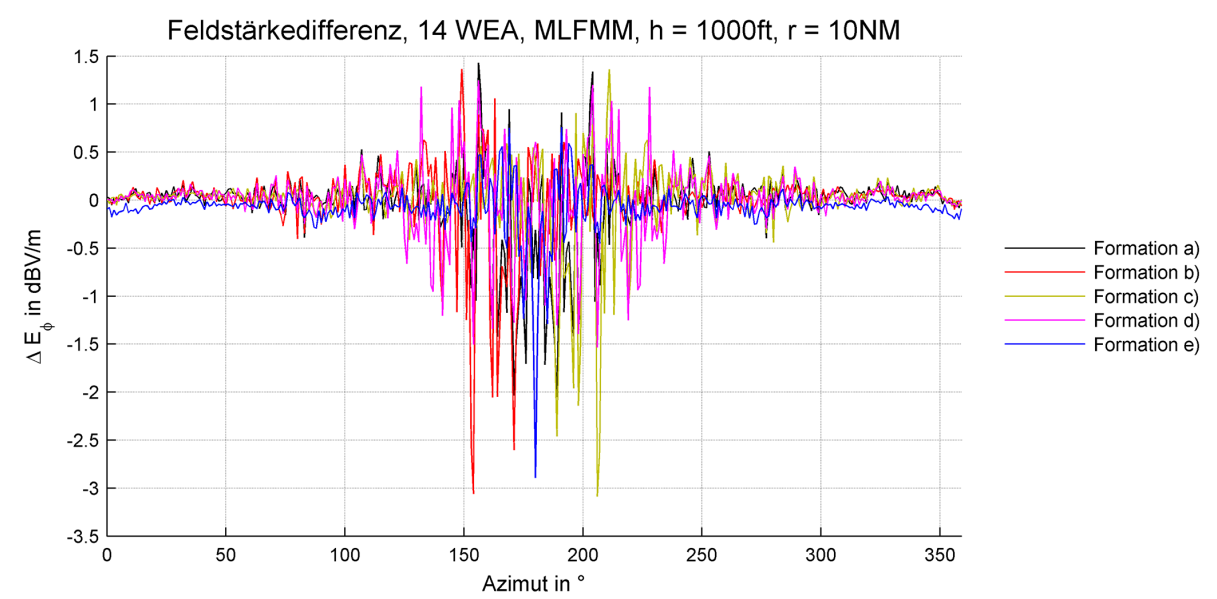

Abb. 16. Feldstärkedifferenzen der 5. Kreisbahn bei Variation der WEA-Park-Formation. 


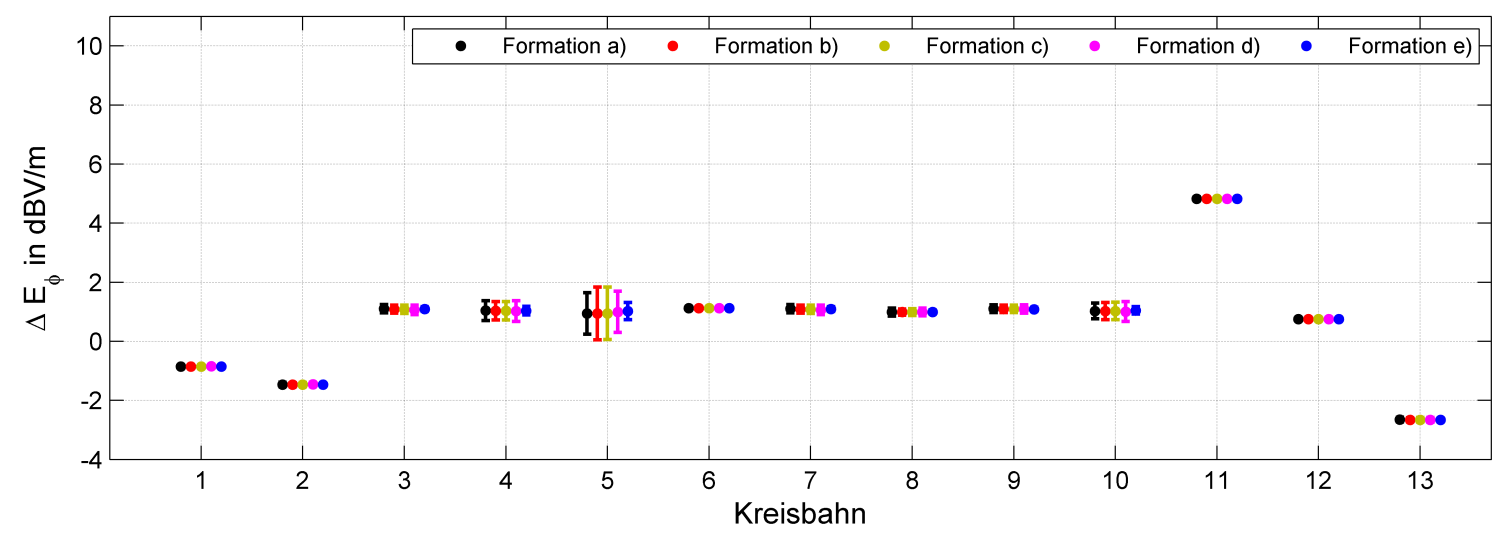

Abb. 17. PO-Simulationsergebnisse der Kreisbahnen 1 bis 13 bei Variation der WEA-Park-Formation.

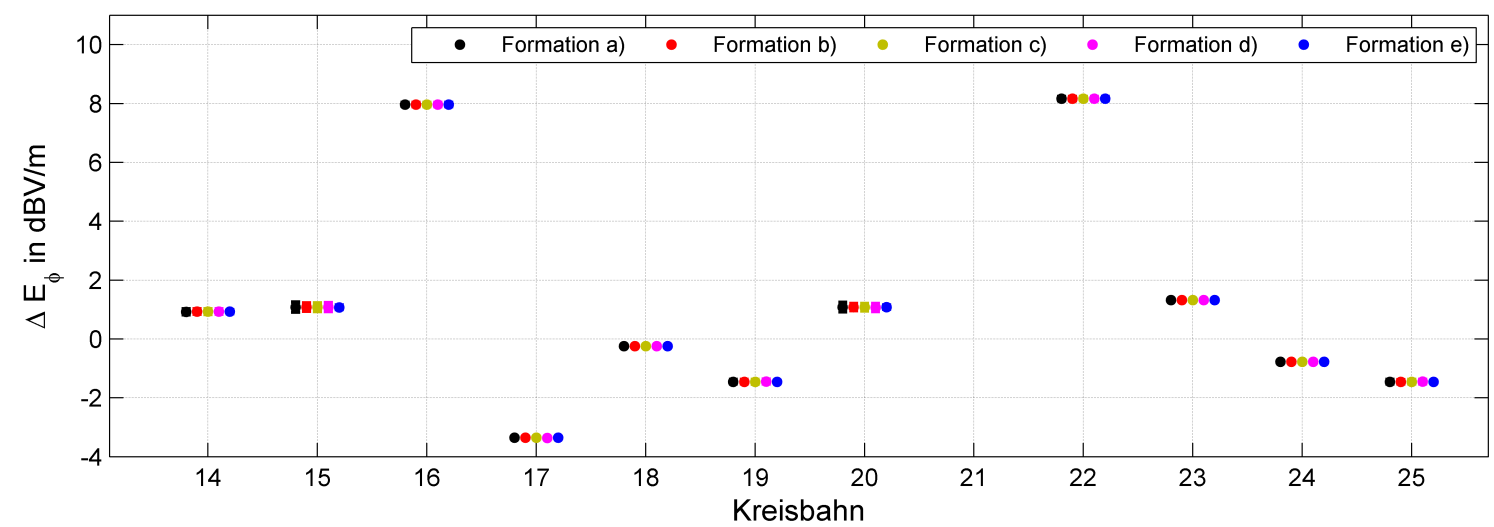

Abb. 18. PO-Simulationsergebnisse der Kreisbahnen 14 bis 25 bei Variation der WEA-Park-Formation. Die Werte der 21. Kreisbahn sind aus Darstellungsgründen nicht abgebildet $\left(\left|M_{21}\right| \leq 26,68 \mathrm{dBV} \mathrm{m}^{-1} ;\left|S_{21}\right| \leq 0,41 \mathrm{dBV} \mathrm{m}^{-1}\right)$.

Bei der Simulation der Szenarien mit der Methode PO, deren Ergebnisse in den Abb. 17 und 18 dargestellt sind, sind diese Auffälligkeiten nicht zu verzeichnen, wobei die Beträge der Mittelwerte insgesamt um ein vielfaches höher Ausfallen und dadurch sowohl die Unterschiede zwischen den einzelnen WEA-Parkformationen als auch die Standardabweichungen der einzelnen Kreisbahnen in dieser Größenordnung nicht mehr aufgelöst werden. Lediglich bei der 5. Kreisbahn sind höhere Standardabweichungen zu verzeichnen, die auf die erhöhten Störungen der Feldstärke im Windschatten der WEA zurückzuführen sind.

\section{Fazit}

Die hier vorgestellten Untersuchungen haben gezeigt, dass die größten Änderungen der Feldstärke außerhalb des ,,cone of silence" mit Absolutwerten von typischerweise unter $3 \mathrm{dBV} \mathrm{m}^{-1}$ direkt hinter dem WEA-Park in seinem elektromagnetischen Schatten auftreten. In den sonstigen Bereichen liegen diese typischerweise bei unter $1 \mathrm{dBV} \mathrm{m}^{-1}$. Bei Variation der WEA-Parameter wurde der größte Einfluss auf die
Feldstärkedifferenzen von der Anzahl der WEA und der Formation des WEA-Parks verursacht. Die sonstigen untersuchten Parameter, wie Turmhöhe, Rotordurchmesser und Rotorwinkel zeigten keine nennenswerten Tendenzen. Die Simulationsmethode PO lieferte bei deutlich geringerem Ressourcenbedarf Ergebnisse, die sich vor allem bei einfachen geometrischen Verhältnissen als Richtwert verwenden lassen. Durch die teilweise relativ hohen Abweichungen von über $10 \mathrm{dBV} \mathrm{m}^{-1}$ lässt sich die Zulässigkeit der Simulationsmethode PO bei der betrachteten Frequenz von $112 \mathrm{MHz}$ für die Berechnung von exakten Ergebnissen jedoch nicht bestätigen. 
Danksagung. Diese Arbeit wurde teilweise unterstützt vom Verbundprojekt WERAN. Gefördert durch das Bundesministerium für Wirtschaft und Energie aufgrund eines Beschlusses des Deutschen Bundestages (FKZ: 0325644B).

Des Weiteren wurden die Ergebnisse dieser Arbeit teilweise mit der Software FEKO erstellt (http://feko.info).

Wir bedanken uns für die Unterstützung durch die Deutsche Forschungsgemeinschaft und den Open Access Publishing Fund der Leibniz Universität Hannover.

Edited by: T. Schrader

Reviewed by: two anonymous referees

\section{Literatur}

Gallardo-Hernando, B., Munoz-Ferreras, J. M., Perez-Martinez, F., und Aguado-Encabo, F.: Wind turbine clutter observations and theoretical validation for meteorological radar applications, IET Radar Sonar. Nav., 5, 111-117, 2011.

Jakobus, U. und Meyer, F. J. C.: A hybrid physical optics/method of moments numerical technique: theory, investigation and application, IEEE AFRICON 4th, Stellenbosch, South Africa, 27-27 September, no. 1, 282-287, 1996.

van Tonder, J. J. und Jakobus, U.: Fast multipole solution of metallic and dielectric scattering problems in FEKO, IEEE/ACES International Conference on Wireless Communications and Applied Computational Electromagnetics, Honolulu, HI, USA, 3-7 April 2005, 511-514, 2005.

Wakabayashi, R., Kawakami, H., Sato, G., Amano, T., und Suzuki, Y.: Analysis of course errors on CVOR antennas (including effects of mutual coupling between elements), IEEE Transactions on Vehicular Technology, 47, 392-405, 1998.

Yamamoto, K. und Nagaoka, M.: A bearing error in the VHF OmniRange due to sea surface reflection, Ministry of Transport, Japan, Sixth International Conference on Antennas and Propagation, Coventry, UK, 4-7 April 1989, no. 2, 181-185, 1989. 